\title{
Factores de riesgo perinatal
}

\section{Perinatal Risk Factors}

\author{
Rosa M. Guevara-Cabrera ${ }^{1}$, Sandra I. De la Cruz-Angeles ${ }^{2}$, Paola Del Villar-Santos ${ }^{3}$, \\ Anayeli Hernández-Hernández ${ }^{4}$, Rosa M. Baltazar-Téllez ${ }^{5}$, Roció B. Mayorga-Ponce ${ }^{6}$
}

\begin{abstract}
:
During pregnancy the product is lodged in the uterine cavity from fertilization to birth, but not every pregnancy is guaranteed to be achieved under the best conditions, here a set of homeostatic mechanisms perform their activity to ensure safety and comfort.

Risk factors are considered all those characteristics that expose the binomial, to suffer an injury or disease, in the gravid period, there are multiple risk factors of the microenvironment, matroenvironment and macroenvironment that can affect the fetus and modify its development and physiology.

Some factors may be related to the environment, to the functional state of the mother and to her intrauterine environment. (Mondragón Castro \& Mondagrón Alcocer, 2006)
\end{abstract}

Keywords:

Pregnancy, perinatal factors, fetus, macroenviroment, matroenviroment, microenviroment

\section{Resumen:}

Durante el embarazo el producto se aloja en la cavidad uterina desde la fecundación hasta el nacimiento, pero no todo embarazo tiene la garantía de lograrse en las mejores condiciones, existen un conjunto de mecanismos homeostáticos que desempeñan su actividad para garantizar seguridad y confort.

Los factores de riesgo se consideran todas aquellas características que exponen al binomio, a sufrir una lesión o enfermedad. En el periodo de gravidez, existen múltiples factores de riesgo del microambiente, matroambiente y macroambiente que pueden afectar al feto y modificar su desarrollo y fisiología.

Algunos factores pueden ser relacionados al entorno, a su estado físico funcional de la madre y a su ambiente intrauterino. (Mondragón Castro \& Mondagrón Alcocer, 2006)

Palabras Clave:

Embarazo, factores perinatales, feto, macroambiente, matroambiente, microambiente

\footnotetext{
${ }^{1}$ Autor de correspondencia. Universidad Autónoma del Estado de Hidalgo. Pachuca de Soto. México. Email: rosy_guevara29@hotmail.com

${ }^{2}$ Universidad Autónoma del Estado de Hidalgo. Pachuca de Soto. México. Email: Le.isabel_angeles@hotmail.com

${ }^{3}$ Universidad Autónoma del Estado de Hidalgo. Pachuca de Soto. México. Email: paodelvillar21@gmail.com

${ }^{4}$ Universidad Autónoma del Estado de Hidalgo. Pachuca de Soto. México. Email: hana.gem8@ gmail.com

${ }^{5}$ Universidad Autónoma del Estado de Hidalgo. Pachuca de Soto. México. Email: baltazartellez@yahoo.com.mx

${ }^{6}$ Universidad Autónoma del Estado de Hidalgo. Pachuca de Soto. México. Email: robel62@ hotmail.com
} 


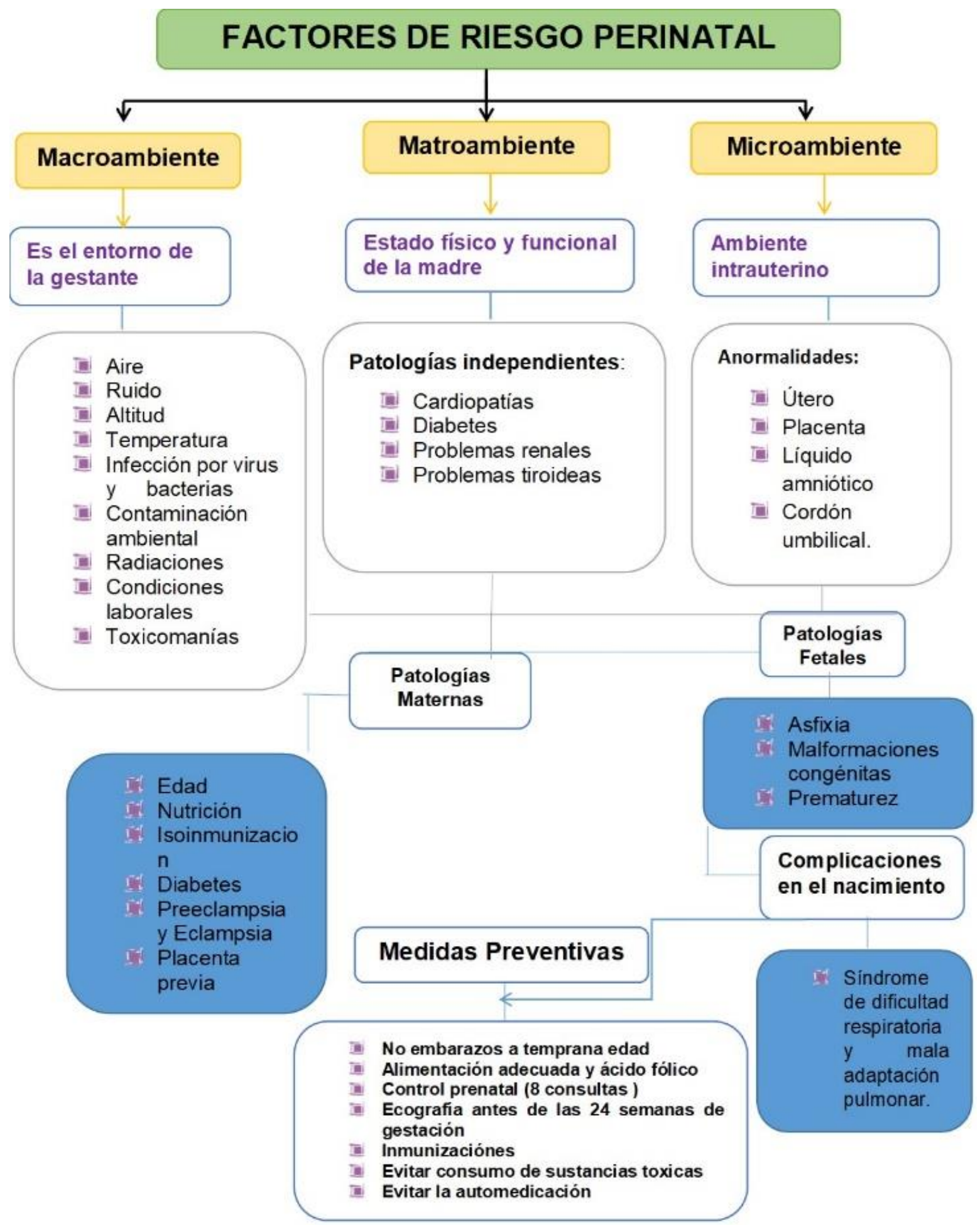

\section{Referencia}

1. Mondragón Castro, H., \& Mondagrón Alcocer, H. (2006). Gineco-Obstetricia de la niñez a la senectud (segunda edición ed.). México: Editorial Trillas.

2. NOM-007-SSA2-2016 PARA LA ATENCIÓN DE LA MUJER DURANTE EL EMBARAZO, PARTO Y PUERPERIO, Y DE LA PERSONA RECIÉN NACIDA 